\title{
Concurrence of symmetrical peripheral gangrene and venous limb gangrene following polytrauma: a case report
}

Jih Huei Tan ${ }^{1,2}$, Yuzaidi Mohamad ${ }^{2}$, Chor Lip Henry Tan ${ }^{1,2}$, Mahazir Kassim ${ }^{3}$ and Theodore E. Warkentin ${ }^{4, *^{*}}$

\begin{abstract}
Background: Symmetrical peripheral gangrene is characterized as acral (distal extremity) ischemic limb injury affecting two or more extremities, without large vessel obstruction, typically in a symmetrical fashion. Risk factors include hypotension, disseminated intravascular coagulation, and acute ischemic hepatitis ("shock liver"). In contrast, venous limb gangrene is characterized by acral ischemic injury occurring in a limb with deep vein thrombosis. Both symmetrical peripheral gangrene and venous limb gangrene present as acral limb ischemic necrosis despite presence of arterial pulses. The coexistence of symmetrical peripheral gangrene and venous limb gangrene is rare, with potential to provide pathophysiological insights.
\end{abstract}

Case presentation: A 42-year-old Chinese man presented with polytrauma (severe head injury, lung contusions, and right femur fracture). Emergency craniotomy and debridement of right thigh wound were performed on presentation. Intraoperative hypotension secondary to bleeding was complicated by transient need for vasopressors and acute liver enzyme elevation indicating shock liver. Beginning on postoperative day 5 , he developed an acute platelet count fall (from 559 to $250 \times 10^{9} / \mathrm{L}$ over 3 days) associated with left iliofemoral deep vein thrombosis that evolved to bilateral lower limb ischemic necrosis; ultimately, the extent of limb ischemic injury was greater in the left (requiring belowknee amputation) versus the right (transmetatarsal amputation). As the presence of deep vein thrombosis is a key feature known to localize microthrombosis and hence ischemic injury in venous limb gangrene, the concurrence of unilateral lower limb deep vein thrombosis in a typical clinical setting of symmetrical peripheral gangrene (hypotension, proximate shock liver, platelet count fall consistent with disseminated intravascular coagulation) helps to explain asymmetric limb injury - manifesting as a greater degree of ischemic necrosis and extent of amputation in the limb affected by deep vein thrombosis - in a patient whose clinical picture otherwise resembled symmetrical peripheral gangrene.

Conclusions: Concurrence of unilateral lower limb deep vein thrombosis in a typical clinical setting of symmetrical peripheral gangrene is a potential explanation for greater extent of acral ischemic injury in the limb affected by deep vein thrombosis.

Keywords: Amputations, Deep vein thrombosis, Symmetrical peripheral gangrene, Venous limb gangrene

\footnotetext{
* Correspondence: twarken@mcmaster.ca

${ }^{4}$ Department of Pathology and Molecular Medicine, McMaster University,

Hamilton, ON, Canada

${ }^{5}$ Hamilton Regional Laboratory Medicine Program, Hamilton General

Hospital, Room 1-270B, 237 Barton St. East, Hamilton, ON L8L 2X2, Canada

Full list of author information is available at the end of the article
}

(c) The Author(s). 2018 Open Access This article is distributed under the terms of the Creative Commons Attribution 4.0 International License (http://creativecommons.org/licenses/by/4.0/), which permits unrestricted use, distribution, and reproduction in any medium, provided you give appropriate credit to the original author(s) and the source, provide a link to the Creative Commons license, and indicate if changes were made. The Creative Commons Public Domain Dedication waiver (http://creativecommons.org/publicdomain/zero/1.0/) applies to the data made available in this article, unless otherwise stated. 


\section{Background}

Symmetrical peripheral gangrene (SPG) is defined as distal ischemic damage in two or more extremities, without large vessel obstruction or vasculitis, typically in a largely symmetrical fashion [1, 2]. Disseminated intravascular coagulation (DIC), usually occurring in the setting of hypotension and shock (septic, cardiogenic, hypovolemic), is the major contributing factor [1-3]. Recently, a role for preceding acute ischemic hepatitis ("shock liver") has been identified in approximately $90 \%$ of patients with SPG; the relevant concept is that acute liver injury predisposes to microthrombosis, and resulting ischemic limb injury, through decreased hepatic production of the key natural anticoagulants, protein $C$ and antithrombin [1, 4]. SPG is associated with a mortality rate of up to $40 \%$ [5].

In comparison with SPG, venous limb gangrene (VLG) results from a different pathogenesis, as by definition it involves unilateral deep vein thrombosis (DVT) in the limb affected by acral (distal extremity) ischemic necrosis. Patients typically have associated DIC, and the concept is that large vein thrombosis predisposes to microthrombosis in the same limb due to decreased blood flow and/or direct contiguous extension of thrombosis [1]. Since DVT most often occurs in one limb, VLG usually presents with unilateral limb ischemic necrosis, whereas by definition SPG usually affects two (or four) limbs in a mainly symmetrical fashion [1]. However, common to both VLG and SPG, patients present with the typical clinical picture of "ischemic limb gangrene with pulses" [1].

Usually, SPG occurs without associated DVT [1]. However, we present a case of critical illness complicated by hypotension, shock liver, and thrombocytopenia, where the patient developed SPG together with acute left iliofemoral DVT. The patient exhibited a disproportionate degree of injury, as shown by the higher level of amputation required in the limb that was also affected by DVT. The clinical picture indicates concurrence of SPG and VLG and suggests that the presence of DVT can modulate the clinical picture of SPG. To the best of our knowledge, a similar case has not been reported in the literature.

\section{Case presentation}

A 42-year-old Chinese man riding a motorbike sustained blunt trauma following collision with a lorry. He had decreased level of consciousness and a nosebleed when found lying at the roadside. On arrival at the emergency department, his Glasgow Coma Scale (GCS) was E2V2M4, right pupil was $4 \mathrm{~mm}$ and left pupil was $2 \mathrm{~mm}$ with sluggish reactions. His blood pressure (BP) was 169/ $126 \mathrm{mmHg}$, heart rate 89 beats/minute, and arterial oxygen saturation $\left(\mathrm{SaO}_{2}\right)$ was $80 \%$ (room air). He was intubated and resuscitated following principles of Advanced Trauma Life Support. A computed tomography (CT) scan of his brain showed right frontal intraparenchymal bleeding $(3.5 \times 1.5 \times 2.6 \mathrm{~cm})$, right cerebral sulci effacement, 0 . $9 \mathrm{~cm}$ midline shift, multiple extradural hemorrhages of $1 \mathrm{~cm}$ diameter, and multiple small contusions in bilateral frontal and right temporal regions. A thoracic and abdominal contrast CT scan showed bilateral lung contusions with multiple rib fractures. Pelvic and right femur Xrays showed fractures of the right neck of femur and midshaft with open wound (Gustilo grade two). Bilateral pedal pulses were palpable.

Within 3 hours post-trauma, emergency decompressive craniectomy and right femur wound debridement were performed. Intraoperatively, his BP dropped to $80 / 50 \mathrm{mmHg}$ due to bleeding from his right femur and craniectomy, and noradrenaline infusion was started (highest dose, $0.16 \mathrm{mcg} / \mathrm{kg}$ per minute). Postoperatively, his alanine aminotransferase level measured $182 \mathrm{U} / \mathrm{mL}$ (reference range, 10-40) and aspartate aminotransferase level measured $405 \mathrm{U} /$ $\mathrm{mL}$ (reference range, 10-40), consistent with hypotension-related shock liver in this clinical context.

Estimated intraoperative total blood loss was 11 . Two units of packed cells were transfused. Postoperatively, he was ventilated in an intensive care unit, and intravenously administered fentanyl and propofol were maintained for 24 hours. Following stoppage of sedation, noradrenaline was weaned off (on postoperative day 2). However, his subsequent GCS recovery was poor (E1VtM4). Unfractionated heparin (5000 IU twice daily by subcutaneous injection) was commenced on the third day for DVT prophylaxis, as risk of bleeding from injured organs was deemed to be low at that time.

On day 5, he developed bilateral lower limb swelling extending into both thighs, more marked on his left thigh. Bluish discoloration of all toes was noted with presence of bilateral pedal Doppler signals. His fingers were normal. Duplex scanning of the deep veins of his lower limbs showed left external iliac and femoral vein thrombosis, with no DVT on the right side. CT angiography revealed good opacification of arteries to the small vessels distal to both ankles, consistent with bilateral distal microvascular thrombosis; that is, the radiological picture was consistent with SPG. Low-dose heparin thromboprophylaxis was replaced by therapeutic-dose intravenously administered heparin upon diagnosis of DVT of his left lower limb; at the same time, hemodialysis was commenced.

His platelet count fell abruptly from $559 \times 10^{9} / \mathrm{L}$ on the fifth hospital day (measured shortly before onset of limb ischemia) to 250 on the eighth day, a decline of approximately $55 \%$ (Fig. 1). Progressive acral limb ischemia occurred during this time period. The platelet 

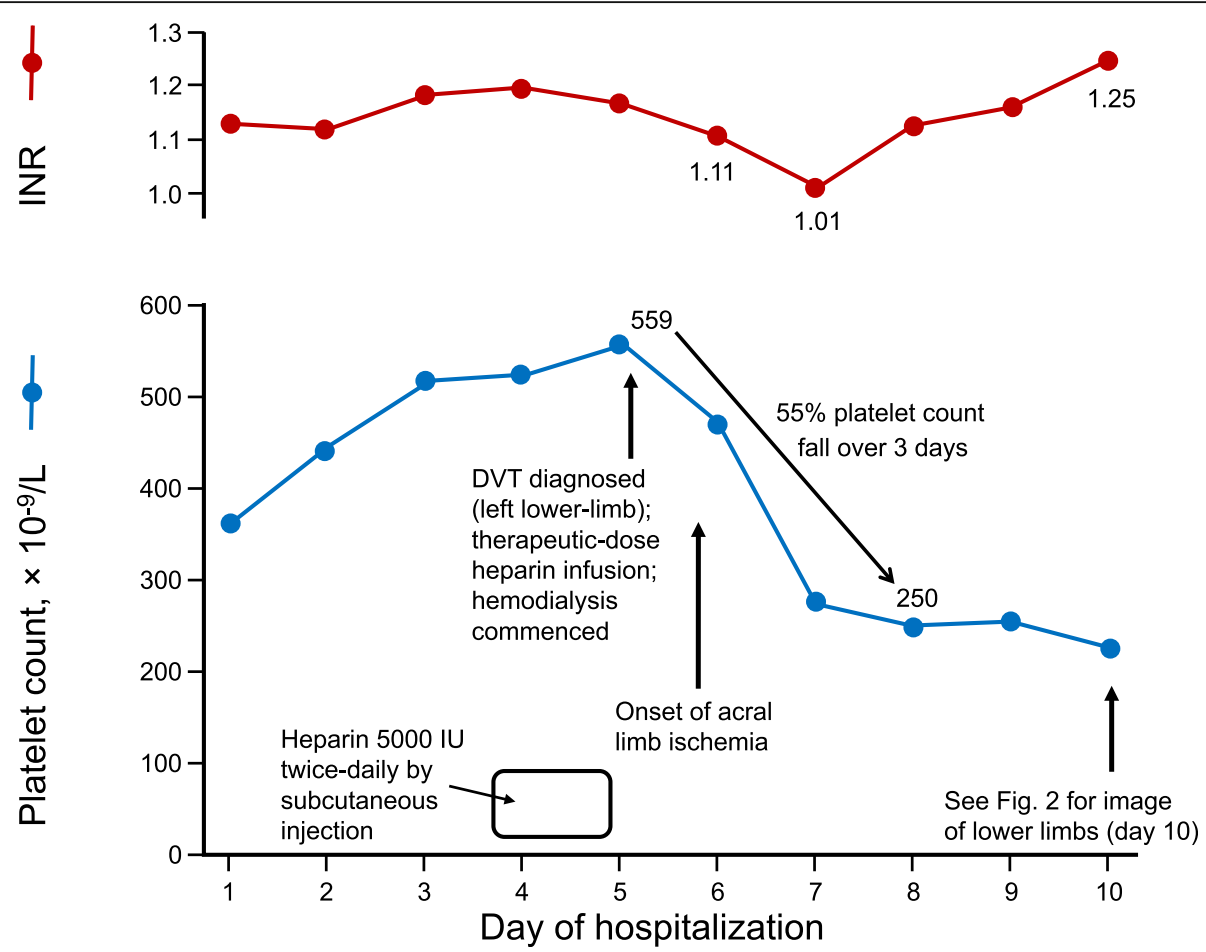

Fig. 1 Changes in platelet count and international normalized ratio from hospital day 1 to day 10. The beginning of the 55\% platelet count decline coincided with the onset of progressive acral ischemia. DVT deep vein thrombosis, INR international normalized ratio

count fall (starting on the third day of heparin therapy) was deemed too soon to indicate immune heparininduced thrombocytopenia (HIT) on clinical grounds (although testing for HIT antibodies was not locally available).

At the time of onset of limb ischemia (day 6 of hospitalization), blood testing showed: hemoglobin, $75 \mathrm{~g} / \mathrm{L}$; white cell count, $12.7 \times 10^{9} / \mathrm{L}$; C-reactive protein $(\mathrm{CRP})$, $169.7 \mathrm{mg} / \mathrm{l}$ (reference range, <10); erythrocyte sedimentation rate, $61 \mathrm{~mm}$ (reference range, 0-22); prothrombin time, 11.6 (reference range, 11.5-15.5 seconds); international normalized ratio (INR), 1.11 (reference range, $<1.4$ ); and activated partial thromboplastin time, 43.3 seconds (reference range, 30-40 seconds). He had acute renal failure with urea of $43.8 \mathrm{mmol} / \mathrm{L}$ (reference range, 2.8-8.1) and serum creatinine of $357 \mu \mathrm{mol} / \mathrm{L}$ (reference range, 62-106), which resolved after a week. Blood cultures were all negative.

Thrombectomy was done by the vascular surgeon via femoral vein. Two days post-thrombectomy, left iliofemoral venous thrombosis recurred, and repeat thrombectomy was performed. Despite heparin treatment and repeat thrombectomies, toe ischemia progressed to more extensive lower limb ischemic gangrene (Fig. 2 showing clinical appearance on day 10). Right transmetatarsal and left below-knee amputations were performed. However, he died during his sixth week of hospitalization due to pneumonia and infected bed sore.

\section{Discussion and conclusions}

SPG is a clinical syndrome with largely symmetrical acral (distal extremity) necrosis despite palpable or Doppler-identifiable arterial pulses, more often involving the lower limbs; the upper limbs are additionally affected in only one-third of cases [1]. VLG is defined as acral necrosis of an extremity in the setting of an acute DVT affecting the same limb [1]. Both entities share a similar pathophysiological feature in that there is microthrombosis associated with a DIC state and natural anticoagulant failure $[1,6]$.

SPG has been reported in a variety of clinical scenarios. Its common associations are septic and cardiogenic shock [1]. Other reported clinical settings of SPG include malaria [7], dengue fever [8], post-cardiac surgery [9], pancreatitis [10], post-partum [11], and following snake bite [12]. All of these conditions are often associated with a proinflammatory state; the inflammatory cytokines trigger DIC and potential for microthrombosis. Low flow state secondary to hypotension or cardiac failure together with acquired protein $\mathrm{C}$ and antithrombin depletion further perpetuate acral microthrombosis [1]. Our polytrauma patient had several risk factors for SPG, including multiple injuries inducing a proinflammatory response (high CRP level) and acute hepatic dysfunction (shock liver) as a contributing factor in subsequent natural anticoagulant depletion (although we were not able 


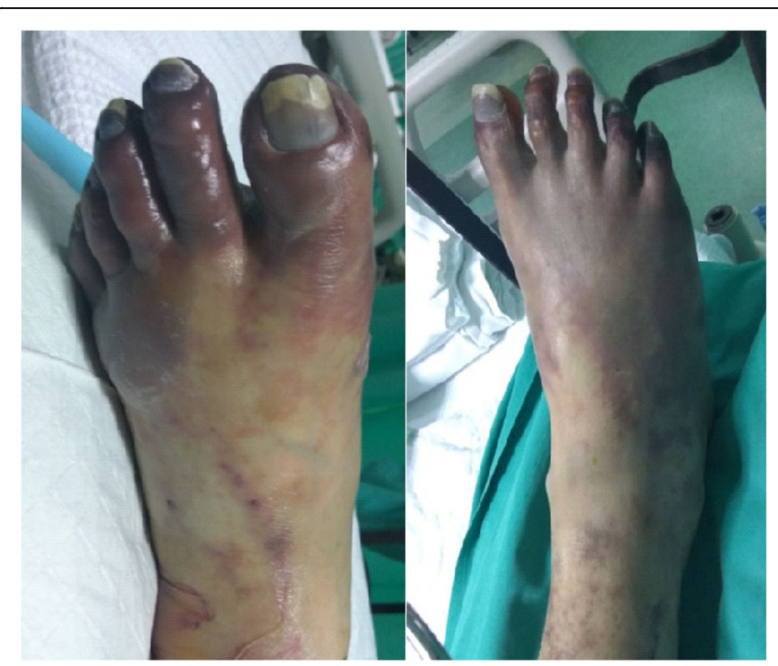

Fig. 2 Right foot and left foot of this patient, showing that all his toes are gangrenous (photo taken at day 10)

to measure these natural anticoagulant factors in our hospital in Malaysia). Further depletion of protein $C$ has been reported to occur in the setting of polytrauma [13].

The concurrent presence of DVT in one affected limb adds additional complexity to the case, as DVT complicating SPG is rare. Although both entities can be associated with DIC, the underlying triggers of DIC tend to differ. Patients with VLG usually present with unilateral proximal DVT in association with an underlying hypercoagulability state [1], such as HIT [14, 15] or cancerrelated consumptive coagulopathy [16], and often with protein $C$ depletion induced by warfarin $[14,16]$. A case of VLG after initiation of rivaroxaban has also been reported [17].

In comparison with previously reported cases of SPG, the presence of DVT with VLG in our current case is unusual. He did not have an underlying malignancy, warfarin intake, or elevated INR. However, our patient did develop an abrupt platelet count fall from 559 to 250 that coincided with the development of ischemic limb injury. Although we cannot definitively rule out the possibility of HIT (as testing for HIT antibodies could not be performed), the early timing of onset of thrombocytopenia in relation to start of heparin prophylaxis makes this drug reaction unlikely [18]. However, the abrupt onset of platelet count fall, presumably reflecting a degree of platelet activation, irrespective of cause, probably played an important role in predisposing our patient to microvascular thrombosis.

There is no single effective therapy to treat both SPG and VLG. Treatment for SPG includes avoidance/dose-reduction of vasopressors and administration of an anticoagulant such as heparin (if HIT is not present). Other reported measures include sympathetic blockade, intravenously administered vasodilators, local injection or intravenous infusion of alpha-blockers, and intravenously administered prostaglandins, but with variable (usually minimal) degree of success. Other maneuvers to prevent DVT/VLG include intermittent calf compression, extremity elevation, fluid resuscitation, and avoidance of warfarin. Thrombolysis with urokinase is another potential therapy. If thrombolysis is contraindicated (as in our patient case), surgical venous thrombectomy plus or minus distal arteriovenous fistula is an option to promote recanalization [19]; if compartment syndrome develops, fasciotomy is indicated.

\section{Conclusion}

The occurrence of DVT in a patient with post-trauma SPG was associated with greater ischemic limb injury in the limb affected by the DVT. Thus, our patient appears to have developed SPG (critical illness, hypotension resulting in shock liver, hypercoagulability state with dropping platelet count, and bilateral lower limb ischemic injury despite presence of arterial pulses) together with VLG (that is, presence of DVT in a limb with ischemic necrosis despite presence of arterial pulses). He developed a greater degree of ischemic injury in the limb affected by DVT, indicating that concomitant DVT can modulate the clinical course of SPG.

\section{Abbreviations \\ BP: Blood pressure; CRP: C-reactive protein; CT: Computed tomography; DIC: Disseminated intravascular coagulation; DVT: Deep vein thrombosis; GCS: Glasgow Coma Scale; HIT: Heparin-induced thrombocytopenia; INR: International normalized ratio; IV: Intravenous; SPG: Symmetrical peripheral gangrene; VLG: Venous limb gangrene}

\section{Funding}

This case report was self-funded.

\section{Availability of data and materials}

Data sharing not applicable to this article as no datasets were generated or analyzed during the current report.

\section{Authors' contributions}

JHT was involved in collecting information, and drafting and reporting of the work described in the article. MK, CLHT, and YM were involved in the drafting of this manuscript. TEW helped to interpret the clinical and laboratory information, proposed a role for DVT in modulating the clinical presentation of SPG, and critically reviewed the manuscript for content. All authors read and approved the final manuscript.

\section{Ethics approval and consent to participate}

Ethical approval was granted by Ministry of Health Malaysia Medical Research Ethics Committee.

\section{Consent for publication}

Written consent was obtained from the patient's next-of-kin for publication of this case report and accompanying images. A copy of the written consent is available for review by the Editor-in-Chief of this journal on request.

\section{Competing interests}

T.E. Warkentin reports consulting fees from Aspen Global, research support and consulting fees from W.L. Gore, research support and consulting fees from Instrumentation Laboratory, research support and consulting fees from Medtronic Diabetes, consulting fees from Octapharma, royalties from Informa (Taylor \& Francis), and consulting fees relating to medical-legal testimony. The other authors declare that they have no competing interests. 


\section{Publisher's Note}

Springer Nature remains neutral with regard to jurisdictional claims in published maps and institutional affiliations.

\section{Author details}

'Department of General Surgery, Pusat Perubatan Universiti Kebangsaan Malaysia, Cheras, Malaysia. ${ }^{2}$ Department of General Surgery, Hospital Sultanah Aminah, Johor Bahru, Malaysia. ${ }^{3}$ Department of Anaesthesiology and Intensive Care, Hospital Sultanah Aminah, Johor Bahru, Malaysia. ${ }^{4}$ Department of Pathology and Molecular Medicine, McMaster University, Hamilton, ON, Canada. ${ }^{5}$ Hamilton Regional Laboratory Medicine Program, Hamilton General Hospital, Room 1-270B, 237 Barton St. East, Hamilton, ON L8L 2X2, Canada.

Received: 7 December 2017 Accepted: 9 April 2018

Published online: 19 May 2018

\section{References}

1. Warkentin TE. Ischemic limb injury with pulses. N Engl J Med. 2015;373(7): 642-55.

2. Sharma BD, Kabra SR, Gupta B. Symmetrical peripheral gangrene. Trop Dr. 2004:34(1):2-4

3. Ghosh SK, Bandyopadhyay D, Ghosh A. Symmetrical peripheral gangrene: a prospective study of 14 consecutive cases in a tertiary-care hospital in eastern India. J Eur Acad Dermatol Venereol. 2010;24(2):214-8.

4. Warkentin TE. Heparin-induced thrombocytopenia in critically ill patients Semin Thromb Hemost. 2015;41(1):49-60.

5. Phan PN, Acharya $V$, Parikh D, Shad A. A rare case of symmetrical four limb gangrene following emergency neurosurgery. Int J Surg Case Rep. 2015;16: $15-8$.

6. Hotchkiss RS, Levy JH, Levi M. Sepsis-induced disseminated intravascular coagulation, symmetrical peripheral gangrene, and amputations. Crit Care Med. 2013;41(10):e290-1.

7. Abdali N, Malik AM, Kamal A, Ahmad M. Symmetrical peripheral gangrene due to Plasmodium falciparum malaria. BMJ Case Rep. 2014;26:2014. https://doi.org/10.1136/bcr-2014-204268.

8. Patel ML, Sachan R, Verma A, Shyam R. Symmetrical peripheral gangrene: Unusual complication of dengue fever. Adv Biomed Res. 2016;5:154 https://doi.org/10.4103/2277-9175.188940.

9. Reed RC. Symmetrical peripheral gangrene in four pediatric cardiac surgery patients receiving extracorporeal membrane oxygenation. Pediatr Dev Pathol. 2012;15(3):217-25.

10. Liao CY, Huang SC, Lin CH, Wang CC, Liu MY, Ben RJ, Kuo WH, Lee CC. Successful resolution of symmetrical peripheral gangrene after severe acute pancreatitis: a case report. J Med Case Rep. 2015;9:213. https://doi.org/10. 1186/s13256-015-0688-3.

11. Sharma L, Mehta S, Rajaram S, Aggarwal R, Gupta S, Goel N. Postpartum peripheral symmetrical gangrene: a case report. J Reprod Infertil. 2012;13(2): 117-9.

12. Shastri M, Parikh M, Patel D, Chudasma K, Patell R. Symmetrical peripheral gangrene following snake bite. J Clin Diagn Res. 2014;8(9):MD03-4.

13. Engelman DT, Gabram SG, Allen L, Ens GE, Jacobs LM. Hypercoagulability following multiple trauma. World J Surg. 1996;20(1):5-10.

14. Warkentin TE, Elavathil LJ, Hayward CPM, Johnston MA, Russett J, Kelton JG. The pathogenesis of venous limb gangrene associated with heparininduced thrombocytopenia. Ann Intern Med. 1997;127(9):804-12.

15. Rozati H, Shah SP, Peng YY. Lower limb gangrene postcardiac surgery. BMJ Case Rep. 2013;15:2013. https://doi.org/10.1136/bcr-2012-008362.

16. Warkentin TE, Cook RJ, Sarode R, Sloane DA, Crowther MA. Warfarin-induced venous limb ischemia/gangrene complicating cancer: a novel and clinically distinct syndrome. Blood. 2015;126(4):486-93.

17. Rosenbaum AN, Yu RC, Rooke TW, Heit JA. Venous gangrene and intravascular coagulation and fibrinolysis in a patient treated with rivaroxaban. Am J Med. 2014;127(6):e7-8.

18. Warkentin TE, Kelton JG. Temporal aspects of heparin-induced thrombocytopenia. N Engl J Med. 2001;344(17):1286-92.

19. Laohapensang K, Hanpipat S, Aworn S, Orrapin S. Surgical venous thrombectomy for phlegmasia cerulea dolens and venous gangrene of the lower extremities. J Med Assoc Thail. 2013;96(11):1463-9.

\section{Ready to submit your research? Choose BMC and benefit from:}

- fast, convenient online submission

- thorough peer review by experienced researchers in your field

- rapid publication on acceptance

- support for research data, including large and complex data types

- gold Open Access which fosters wider collaboration and increased citations

- maximum visibility for your research: over $100 \mathrm{M}$ website views per year

At BMC, research is always in progress.

Learn more biomedcentral.com/submissions 